\title{
Circuit
}

Musiques contemporaines

\section{Liste des oeuvres lyriques citées}

Volume 12, numéro 2, 2002

Opéra aujourd'hui

URI : https://id.erudit.org/iderudit/902256ar

DOI : https://doi.org/10.7202/902256ar

Aller au sommaire du numéro

Éditeur(s)

Les Presses de l'Université de Montréal

ISSN

1183-1693 (imprimé)

1488-9692 (numérique)

Découvrir la revue

Citer ce document

(2002). Liste des oeuvres lyriques citées. Circuit, 12(2), 89-100.

https://doi.org/10.7202/902256ar

Ce document est protégé par la loi sur le droit d'auteur. L'utilisation des services d'Érudit (y compris la reproduction) est assujettie à sa politique d'utilisation que vous pouvez consulter en ligne.

https://apropos.erudit.org/fr/usagers/politique-dutilisation/
Cet article est diffusé et préservé par Érudit.

Érudit est un consortium interuniversitaire sans but lucratif composé de l'Université de Montréal, l'Université Laval et l'Université du Québec à Montréal. Il a pour mission la promotion et la valorisation de la recherche. https://www.erudit.org/fr/ 


\section{Liste des œuvres lyriques citées}

ADAMS, John, El Nino (1999-2000)

Création : Theater of Voices, London Voices, Maîtrise de Paris, Deutsches Symphonie-

Orchester Berlin, Kent Nagano (dir.), théâtre du Châtelet, Paris, décembre 2000.

AMY, Gilbert, Le Premier Cercle (1996-99)

Livret d'après Solienitsyne. Création : Opéra national de Lyon, Michel Plasson (dir.),

octobre 1999. Mise en scène : Lukas Hemleb.

ANDRIESSEN, Louis, Rosa, A Horse Drama (1993-94)

Livret de Peter Greenaway. Commande de Pierre Audi pour l'Opéra d'Amsterdam.

Création: Netherlands Opera, Schönberg Ensemble, Asko Ensemble, Reinbert de Leeuw

(dir.), Muziektheater, Amsterdam, novembre 1994. Mise en scène: Peter Greenaway.

ANDRIESSEN, Louis, Writing to Vermeer (1997-99)

Livret de Peter Greenaway. Commande de Pierre Audi pour l'Opéra d'Amsterdam. Création :

Netherlands Opera, Reinbert de Leeuw (dir.), Muziektheater, Amsterdam, décembre 1999.

ANTHEIL, George, Transatlantic (1930)

Livret de George Antheil. Création: Opernhaus, Frankfort, mai 1930.

BARTLEY, Wende, Electric Flesh (2000)

Livret de Thom Sokoloski. Commande d'Autumn Leaf Performance. Création : Festival

Musique-en-scène en collaboration avec le Grame et l'Opéra de Lyon, mars 2000.

BATTISTELLI, Giorgio, Die Entdeckung der Langsamkeit

Livret de M. Klögl. Création : Brême, 1997.

BATTISTELLI, Giorgio, Impressions d'Afrique

Livret de Daniel Loayza et Georges Lavaudant d'après Raymond Roussel.

Création : Teatro Goldoni, Florence, mai 2000.

BAUMANN, Gerd, NYX

Création: Münich (Biennale), 1997.

BERG, Alban, Wozzeck (1917-22)

Livret d'après le drame de Georg Büchner. Création : Erich Kleiber (dir.), Berlin, 1925. 
BERIO, Luciano, Cronaca del luogo

Livret de Talia Pecker-Berio. Commande du Festival Salzbourg pour la soprano Hildegard

Behrens. Création : Sylvain Cambreling (dir.), Felsenreitschule, Salzbourg, juillet 1999.

BERIO, Luciano, Outis (1995-96)

Livret de Dario del Corno et Luciano Berio. Création : La Scala de Milan, octobre 1996.

BIRTWISTLE, Harrison, The Last Supper (1998-99)

Livret de Robin Blaser. Création : Daniel Barenboim (dir.), Staatsoper, Berlin, avril 2000.

BOESMANS, Philippe, La Passion de Gilles (1982)

Livret de Pierre Mertens. Commande de Gérard Mortier. Création : Théâtre Royal de la Monnaie, Bruxelles, octobre 1983.

BOESMANS, Philippe, Reigen (1992)

Livret de Luc Bondy d'après A. Schnitzler. Commande de Bernard Foccroule.

Création: Théâtre Royal de la Monnaie, Bruxelles, mars 1993. Mise en scène :

Luc Bondy.

BOESMANS, Philippe, Wintermärchen (1999)

Livret de Luc Bondy et Marie-Louise Bischofsberger d'après Le Conte d'hiver de

Shakespeare. Création: Théâtre Royal de la Monnaie, Bruxelles, décembre 1999.

CAGE, John, Europeras (1987)

Commande de l'Opéra de Frankfort. Création : Opéra de Frankfort, Schauspielhaus, décembre 1987.

CARTER, Elliott, What's Next? (1997-98)

Livret de Paul Griffiths. Création : Staatsoper, Berlin, 1999, Cité de la Musique, Paris, 2000.

CASKEN, John, God's Liar (1999-2000)

Livret de J. Casken et Emma Warner d'après Father Sergius de L. Tolstoy. Commande du Northern Sinfonia. Création : coproduction de l'Opéra Almeida et du Théâtre de la Monnaie, Ronald Zollman (dir.), Bruxelles, juillet 2001.

CHAN, Ka Nin, The Iron Road (2000)

Livret de Mark Brownell. Commande de CBC Radio, Conseil des arts du Canada, Toronto Arts Council et Conseil des arts de l'Ontario. Création : Tapestry New Opera Works, Wayne Strongman (dir.), Elgin Theater, Toronto, avril 2001.

DAVIES, Peter Maxwell, The Doctor of Myddfai (1995)

Livret de David Pountney. Commande du Welsh National Opera. Création : Welsh National Opera, Richard Armstrong (dir.), Cardiff (Pays de Galles) juillet 1996.

DAVIES, Peter Maxwell, Mr Emmet takes a Walk

Livret de David Pountney. Commande du St. Magnus Festival, Psappha et Muzietheater Transparent. Création : Psappha et Muzietheater Transparent, Etienne Siebens (dir.), St. Magnus Festival, Orkney, juin 2000. 
DAVIES, Peter Maxwell, The Lighthouse (1979)

Livret de Peter Maxwell Davies. Commande du Festival international d'Edimbourg.

Création: The Fires of London, Richard Dufallo (dir.), Murray House Gymnasium,

Edimbourg, septembre 1980.

DAVIES, Peter Maxwell, The No 11 Bus (1984)

Livret de Peter Maxwell Davies. Création : The Fires of London, Günther Baver-Schenk (dir), Queen Elizabeth Hall, Londres, mars 1984.

DEBUSSY, Claude, Pelléas et Mélisande (1902)

Livret de Maurice Maeterlinck. Création : Opéra comique, Paris, avril 1902.

DE PABLO, Luis, La senorita Cristina

Création : José-Ramon Encinar (dir.), Teatro Real, Madrid, janvier 2001.

DONATONI, Franco, Alfred, Alfred (1995)

Commande de T\&M-Nanterre et du festival Musica de Strasbourg. Création : T\&M-

Nanterre, Ed Spanjaard (dir.), festival Musica, Strasbourg, septembre 1998.

DUSAPIN, Pascal, Le Code de Perela

Création: Opéra-Bastille à Paris, prévue pour 2003.

EÖTVÖS, Peter, Le Balcon

Livret de Françoise Morvan avec la collaboration de Peter Eötvös et André Markowicz d'après Le Balcon de Jean Genet. Commande du Festival d'Aix-en-Provence et de I'Ensemble Intercontemporain. Création : Ensemble Intercontemporain, Peter Eötvös (dir.), festival d'Aix-en-Provence, Théâtre de l'Archevêché, juillet 2002. Mise en scène:

Stanislas Nordey.

EÖTVÖS, Peter, Trois Sœurs (1996-97)

Livret de Claus H. Henneberg et Peter Eötvös d'après Tchekhov. Commande de l'Opéra national de Lyon. Création : Opéra national de Lyon, Kent Nagano (dir.), mars 1998.

Mise en scène: Ushio Amagatsu.

ESSYAD, Ahmed, Héloise et Abélard

Livret de Bernard Noël. Commande de l'Opéra national du Rhin et du festival Musica.

Création: Günter Neuhold (dir.), Festival Musica, Mulhouse, octobre 2000.

Mise en scène : Stanislas Nordey.

EVANGELISTA, José, Manuscrit trouvé à Saragosse (2001)

Livret d'Alexis Nouss d'après Jean Potocki. Création : Ensemble de la SMCQ, Walter

Boudreau (dir.), Salle Pierre-Mercure, Montréal, 2001. Mise en scène : Wajdi Mouawad.

EVANGELISTA, José, Plume (1992)

Texte d' Henri Michaux. Soprano et violoncelle.

EVANGELISTA, José, La Porte (1987)

Texte d'Alexis Nouss, à partir de Kafka. Création : Chants libres. Mise en scène :

Joseph St-Gelais. 
FÉNELON, Philippe, Salammbô (1992-94)

Livret de Jean-Yves Masson d'après Gustave Flaubert. Commande de l'Opéra national de Paris avec le concours du Ministère de la Culture. Création : Gary Bertini, (dir.), OpéraBastille, Paris, mai 1998. Mise en scène : Francesca Zambello.

FLOYD, Carlisle, Cold Sassy Tree (2000)

Livret d'après Olive Anne Burns. Commande du Houston Grand Opera et du San Diego Opera. Création : Houston Grand Opera, Wortham Center, Houston, avril 2000.

GLASS, Philip, Einstein on the Beach (1976)

Livret de Robert Wilson et Philip Glass. Création : Philip Glass Ensemble, Festival d'Avignon, 1976.

GLASS, Philip, Satyagraha (1980)

Livret de Philip Glass et Constance Dejong. Texte adapté du Bhagavad-Gita.

Création : Netherlands Opera et l'Utrechts Symfonie Orkest, Christopher Keene (dir.), Rotterdam, 1980.

GLASS, Philip, Akhnaten (1983)

Livret de Philip Glass d'après Shalom Goldman. Commande du Württembergisch

Staatstheater de Stuttgart. Création: Württembergisch Staatstheater,

Dennis Russell Davies (dir.), Stuttgart, 1984.

GOEHR, Alexander, Kantan and Damask Drum (1997-98)

Livret de Alexander Goehr d'après Zeami et Sarugai Koto. Création : Dortmund, 1999.

HALFFTER, Cristóbal, Don Quijote (2000)

Création : Teatro Real, Madrid, 2000.

HARBISON, John, The Great Gatsby (1999)

Livret de John Harbison et M. Horwitz d'après F. Scott Fitzgerald. Commande du Metropolitain Opera.Création : Metropolitain Opera, James Levine (dir.), New York, décembre 1999.

HENZE, Hans Werner, Venus und Adonis (1993-95)

Livret de Hans-Ulrich Treichel. Création : Staatsoper, Münich, 1997.

HÖlLER, York, Der Meister und Margarita (Le Maître et Marguerite) (1984-89)

Livret d'après le roman de Mikhail Boulgakov. Commande de l'Opéra de Paris, Ircam et Hamburgische Staatsoper.Création : Opéra de Paris, Lothar Zagrosek (dir.), PalaisGarnier, Paris, mai 1989. Mise en scène : Hans Nevenfels.

HOLLIGER, Heinz, Schneewitchen (1997-98)

Livret d'après Blanche-Neige de Robert Walser. Commande du Zurich Opera House.

Création : Zürich, 1998.

HÖLSZKY, Adriana, Der unsichtbare Raum/Tragödia (1997)

Création: Kunsthalle, Bonn, 1997. Mise en scène : Wolf Münzer. 
JANÁČEK, Leos, Jenưfa (1894-1903)

D'après la pièce de théâtre de Gabriela Preissová. Création : janvier 1904.

JANÁČEK, Leos, Kátía Kabanová (1920-21)

Livret de Leos Janáček d'après Groza d'Alexander Ostrovsky. Création : 1921.

JOLAS, Betsy, Schliemann (1982-93)

Livret de Bruno Bayen et Betsy Jolas d'après Bayen. Création : Opéra national de Lyon, Kent Nagano (dir.), mai 1995. Mise en scène : Alain Françon.

KULESHA, Gary, Red Emma (1986-95)

Livret de Carol Bolt. Commande du Canadian Opera Company. Création : Canadian Opera Company au Théâtre du Maurier, Toronto, novembre 1995.

LACHENMANN, Helmut, Das Mädchen mit den Schwefelhölzern (1990-96)

D'après le conte $d^{\prime}$ Hans Christian Andersen la Petite Fille aux allumettes. Création :

Staatsoper, Hambourg, 1997.

LARSEN, Libby, Eric Hermannson's Soul (1996)

Livret de Chas Rader-Schieber d'après Willa Cather. Commande du Nebraska Music

Teachers Association. Création : Hal France (dir.), Rose Theater, Omaha, novembre 1998.

LÉON, Tania, Scourge of Hyacinths (1994)

Livret de Tania Léon d'après la pièce de théâtre radiophonique de Wole Soyinka.

Commande de la Biennale de Münich. Création: Biennale de Münich, 1994.

LÉVINAS, Michaël, Go-gol (1996)

Livret de Michaël Lévinas d'après N. V. Gogol. Création : Opéra de Montpellier, festival Musica de Strasbourg, 1996.

LIEBERMANN, Lowell, The Picture of Dorian Gray (1995)

Livret de Lowell Liebermann d'après Oscar Wilde. Commande de l'Opéra de Monte-

Carlo. Création: Steuart Bedford (dir.), Festival Printemps des Arts, avril 1996.

LIEBERMANN, Rolf, Freispruch für Medea (1995)

Livret de Ursula Hass. Création: Hambourg, 1995.

MAHIER, Gustav, Lieder eines fahrenden Gesellen (Chants du Compagnon errant) (1 883-85) Livret de Gustav Mahler. Création : Berlin, 1896.

MANOURY, Philippe, 60 parallèle (1995-97)

Livret de Philippe Manoury, Michel Deutsch et Pierre Strosser. Commande de la Théâtre du Châtelet et Ircam, avec le soutien de la Fondation Beaumarchais, de M. Paul Sacher et de l'Orchestre de Paris. Création : David Robertson (dir.), Théâtre du Châtelet, Paris, 1997. Mise en scène : Pierre Strosser.

MANOURY, Philippe, K (2001)

D'après Le Procès de Kafka. Création : Dennis Russel Davies (dir.), Opéra-Bastille, Paris, mars 2001. Mise en scène: André Engel. 
MATHER, Bruce, La Princesse blanche (1993)

Livret de Renald Tremblay, tiré de Die Weisse Fürstin de Rainer Maria Rilke. Création : Chants Libres, Nouvel Ensemble Moderne, Montréal, 1994. Mise en scène : Guy Beausoleil.

MONTEVERDI, Claudio, La Favola d'Orfeo (1607)

Livret de Allessandro Striggio. Création : Mantua, 1607.

MOZART, Wolfgang Amadeus, II dissoluto punito, ossia II Don Giovanni (1787)

Livret de Lorenzo da Ponte. Création : Prague, 1787.

MUSGRAVE, Thea, Simón Bolivar (1989-92)

Livret de Thea Musgrave. Commande du los Angeles Music Center Opera Association et du Scottish Opera. Création : Virginia Opera, Peter Mark (dir.), Norfolk (Virginie), janvier 1995.

NEUWIRTH, Olga, Bählamms Fest (1997-99)

Livret de Elfriede Jelinek d'après Bählamms Fest de Leonora Carrington. Commande du Wiener Festwochen. Création: Opéra national du Rhin, Johannes Kalitzke (dir.), Wiener Festwochen, Vienne, juin 1999. Mise en scène : Brothers Quay.

NEUWIRTH, Olga, Der Tod und das Mädchen II (1999)

(Hörspiel, pièce théâtre radiophonique) Livret de Elfriede Jelinek.

NEUWIRTH, Olga, Todesraten (Hörspiel) (1997)

Livret de Elfriede Jelinek. Création : 1997.

PETERS, Randolph, Nosferatu (1993)

Livret de Marilyn Gronsdal. Commande du Canadian Opera Company.

Création: Canadian Opera Company, décembre 1993.

PETITGIRARD, Laurent, Joseph Merrick dit Elephant Man (1996-2000)

Livret d'Eric Nonn. Commande de la Fondation Beaumarchais. Création : Opéra d'Ełat de Prague, Laurent Petitgirard (dir.), février 2002. Mise en scène : Daniel Mesguich.

PICHÉ, Jean, Yo soy la desintegracion

Livret de Yan Muckle d'après les journaux de Frida Kahlo. Commande de Chants Libres.

Création : Chants Libres, Espace Go, Montréal, septembre 1997.

PICKER, Tobias, Emmeline (1994-96)

Livret de J. D. McClatchy d'après Judith Rossner. Commande du Santa Fe Opera.

Création : Santa Fe Opera, 1996.

PICKER, Tobias, Fantastic Mr Fox (1998)

Livret de Donald Sturrock d'après Roald Dahl. Commande de la Fondation Roald Dahl.

Création : Los Angeles Opera, Peter Ash (dir.), décembre 1998.

PINTSCHER, Matthias, Thomas Chatterton (1994-97)

Livret de Claus H. Henneberg et Matthias Pintscher d'après H. H. Jahnn. Commande de l'Opéra de Dresde. Création: Dresde, mai 1998.

POULENC, Francis, La Voix humaine (1958)

Livret de Jean Cocteau. Création: Opéra-Comique, salle Favart, Paris, février 1959. 
PREVIN, André, A Streetcar Named Desire (1998)

Livret de Philip Littell d'après la pièce de théâtre de Tennessee Williams.

Création: San Francisco Opera, Andre Previn (dir.), San Francisco, septembre 1998.

PROVOST, Serge, L'Adorable verrotière (1992)

Livret de Claude Gauvreau. Commande de la Société royale du Canada.

Création : Chants Libres, Nouvel Ensemble Moderne, Lorraine Vaillancourt (dir.), salle Claude-Champagne, Montréal, septembre 1992.

RAUTAVAARA, Einojuhani, Aleksis Kivi (1995-96)

Livret de Einojuhani Rautavaara. Création: Savonlinna, 1997.

REICH, Steve, The Cave (1993)

Texte et vidéo de Beryl Korot. Commande du Weiner Festwochen, Holland Festival,

Hebbel Theater (Berlin), Festival D'Automne à Paris, Koninklijike Muntschouwburg,

Théâtre de La Monnaie (Bruxelles), Serious Speakout and South Bank Centre (Londres) et du Next Wave Festival du Brooklyn Academy of Music. Création : Steve Reich Ensemble, Paul Hillier (dir.), Vienna Festival, 1993.

REICH, Steve, The Desert Music (1984)

D'après un texte de Wiliam Carlos Williams. Commande de la Radio ovestallemande (Cologne) et du Brooklyn Academy of Music. Création : Cologne Radio Chorus, Cologne Radio Symphony Orchestra, Peter Eölvös (dir.), Cologne Broadcast House, Allemagne, 1984.

REICH, Steve, It's Gonna Rain (1965)

Pour bande avec la voix de Borther Walter. Création : San Francisco Tape Music Center, 1965.

REICH, Steve, Tehillim (1981)

Commande de la West German Radio (Cologne), South German Radio (Stuttgart), et Rothko Chapel (Houston). Création : West German Radio, Cologne, 1981.

REIMANN, Aribert, Bernarda Albas Haus

D'après Lorca. Création : Bayerischen Staatsoper, Komischen Oper Berlin, Zubin Mehta (dir.), Nationaltheatre, Münich, octobre 2000. Mise en scène : Frank Philipp Schlößmann.

REIMANN, Aribert, Lear (1978)

Livret de Claus $\mathrm{H}$. Henneberg d'après William Shakespeare. Création : Bayerischen Staatsoper, Gerd Albrecht (dir.), Nationaltheater, Münich, 1978.

RIHM, Wolfgang, Die Eroberung von Mexico (1987-91)

Livret de Wolfgang Rihm d'après A. Artaud. Création : Hambourg, 1992.

RIHM, Wolfgang, Séraphin (Versuch eines Theaters für Instrumente/Stimmen/...)

(1 ${ }^{\mathrm{re}}$ version : 1993-94, $2^{\mathrm{e}}$ version : 1993-96) Création : Francfort, 1994.

RUDERS, Poul, The Handmaid's Tale (1997-98)

Livret de Paul Bentley d'après Margaret Atwood. Commande du Royal Danish Opera.

Création : Royal Danish Opera, Michael Schonwandt (dir.), Copenhague, mars 2000. 
SAARIAHO, Kaija, L'Amour de loin (1999-2000)

Livret de Amin Maalouf d'après La Vida brève de Jaufré Rudel. Commande du festival de Salzbourg et du Théâtre du Châtelet à Paris. Création : Kent Nagano (dir.), Festival de Salzbourg, Salzbourg, août 2000.

SAARIAHO, Kaija, Château de l'âme (1996)

Texte tiré de poésie ancienne indienne et égyptienne. Commande de Betty Freeman pour le Festival de Salzbourg. Création : London Philharmonic Orchestra, Esa-Pekka Salonen (dir.), Felsenreitschule, Salzbourg, 1996.

SAEGUSA, Shigeaki, Chushingura (1997)

Livret de Shimada Masahiko. Création : Naoto Otomo (dir.), Tokyo, 1997.

SALLINEN, Aulis, King Lear (Roi Lear) (1997-99)

Livret de Matti Rossi d'après la pièce de William Shakespeare. Commande du Finnish National Opera. Création : Finnish National Opera, Okko Kamu (dir.), Helsinki, septembre 2000.

SALLINEN, Aulis, Kullervo (1986-88)

Livret de Aulis Sallinen. Commande du Finnish National Opera.

Création : Los Angeles Chamber Orchestra, Finnish National Opera Choir,

Ulf Söderblom (dir.), Los Angeles, 1992.

SALLINEN, Aulis, The Palast (Palatsi) (1991-93)

Livret de Hans-Magnus Enzensberger et Irene Dische. Commande du Savonlinna Opera

Festival. Création : Savonlinna Opera Festival, Okko Kamu (dir.), Savonlinna, 1995.

SCELSI, Giancinto, Canti del capricorno (Chants du Capricorne) (1962-72)

Création : Michiko Hirayama (soprano).

SCHAFER, R. Murray, Requiems for the Party Girl (1966)

Livret de R. Murray Schafer. Commande de la CBC pour Phyllis Mailing.

SCIARRINO, Salvatore, Luce mie traditrici (1996-98)

Livret de Salvatore Sciarrino d'après A. Cicognini. Création : Rokokotheater,

Schwetzingen, 1998.

SOTELO, Mauricio, De amore

Livret de Peter Mussbach. Création : Biennale de Münich, 1999.

STRAUSS, Richard, Der Rosenkavalier (Le Chevalier à la rose) (1909-10)

Livret de Hugo von Hofmannsthal. Création : Dresden, 1911.

STRAUSS, Richard, Die Frau Ohne Schatten (La Femme sans ombre) (1914-17)

Livret de Hugo von Hofmannsthal. Création : Vienne, 1919.

STRAUSS, Richard, Elektra (1906-08)

Livret de Hugo von Hofmannsthal d'après Sophocles. Création : Berlin, 1909. 
STRAUSS, Richard, Salomé (1903-05)

Adaptation du drame de Oscar Wilde, traduit en allemand par $\mathrm{H}$. Lachmann.

Création: Ernst von Schuch (dir.), Dresden, 1905.

STRAVINSKY, Igor, L'Histoire du soldat (1918)

Livret de Charles-Ferdinand Ramuz. Création : Ernest Ansermet (dir.), Lausanne, 1918.

STRAVINSKY, Igor, Pulcinella (1919-20)

Livret de Leonide Massine. Création : Opéra de Paris, Ernest Ansermet (dir.), 1920.

SWERTS, Piet, Les Liaisons dangereuses (1996)

Livret de D. Van der Cruysse d'après Laclos. Commande du Flemish Opera.

Création : Flemish Opera, Gand, décembre 1996.

TAN DUN, Marco Polo (1995)

Livret de Paul Griffiths. Commande du Festival Edimbourg. Création : Tan Dun (dir.),

Biennale de Münich, mai 1996.

THIBAUIT, Alain, Lulu, Le Chant du souterrain (2000)

Livret de Yan Muckle. Commande de Chants Libres. Création : Chants Libres, Usine C, Montréal, 2000. Mise en scène: Wajdi Movawad.

THIBAULT, Alain, Ne blâmez jamais les bédouins (1991)

Livret de René-Daniel Dubois. Commande de Chants Libres. Création : Chants Libres, théâtre La Licorne, Montréal, 1991. Mise en scène : Joseph Saint-Gelais.

TURNAGE, Mark-Anthony, Greek (1986-88)

D'après Steven Berkoff, adapté par Mark-Anthony Turnage et J. Moore.

Création : Carl Orff Saal, Biennale de Münich, juin 1988.

TURNAGE, Mark-Anthony, The Country of the Blind (1996-97)

Livret de Clare Venables d'après H. G. Wells. Création : English National Opera, Londres, 1997.

TURNAGE, Mark-Anthony, The Silver Tassie (1997-99)

Livret de Amanda Holden d'après Sean O'Casey. Création : English National Opera, Londres, février 2000.

UNDERHILL, Owen, The Star Catalogues (1994)

Livret de Marc Diamond. Création : Vancouver New Music, Russian Hall, Vancouver, octobre 1994.

VIVIER, Claude, Kopernikus (1979)

Livret de Claude Vivier. Création : Atelier de musique contemporaine et Atelier de jeu scénique de la Faculté de musique de l'Université de Montréal, Lorraine Vaillancourt (dir.), Monument national, mai 1980.

VON BOSE, Hans-Jürgen, Schlachthof 5 (1995)

Livret de Hans-Jürgen Von Bose. Commande de Peter Jonas. Création : Bayerische

Staatsoper, Eike Gramss (dir.), Münich, juillet 1996. Mise scène : Gottfried Pilz. 
WEISENSEL, Neil, The Master's Stroke (2000)

Livret de Michael Cavanagh. Commande du Conseil des Arts du Canada.

Création : octobre 2000.

WIENS, Rainer, Down Here on Earth (1997)

Livret de Victoria Ward. Commande de Autumn Leaf. Création : Autumn Leaf, Thom Sokoloski (dir.), Factory Theatre, Toronto, mars 1997. 


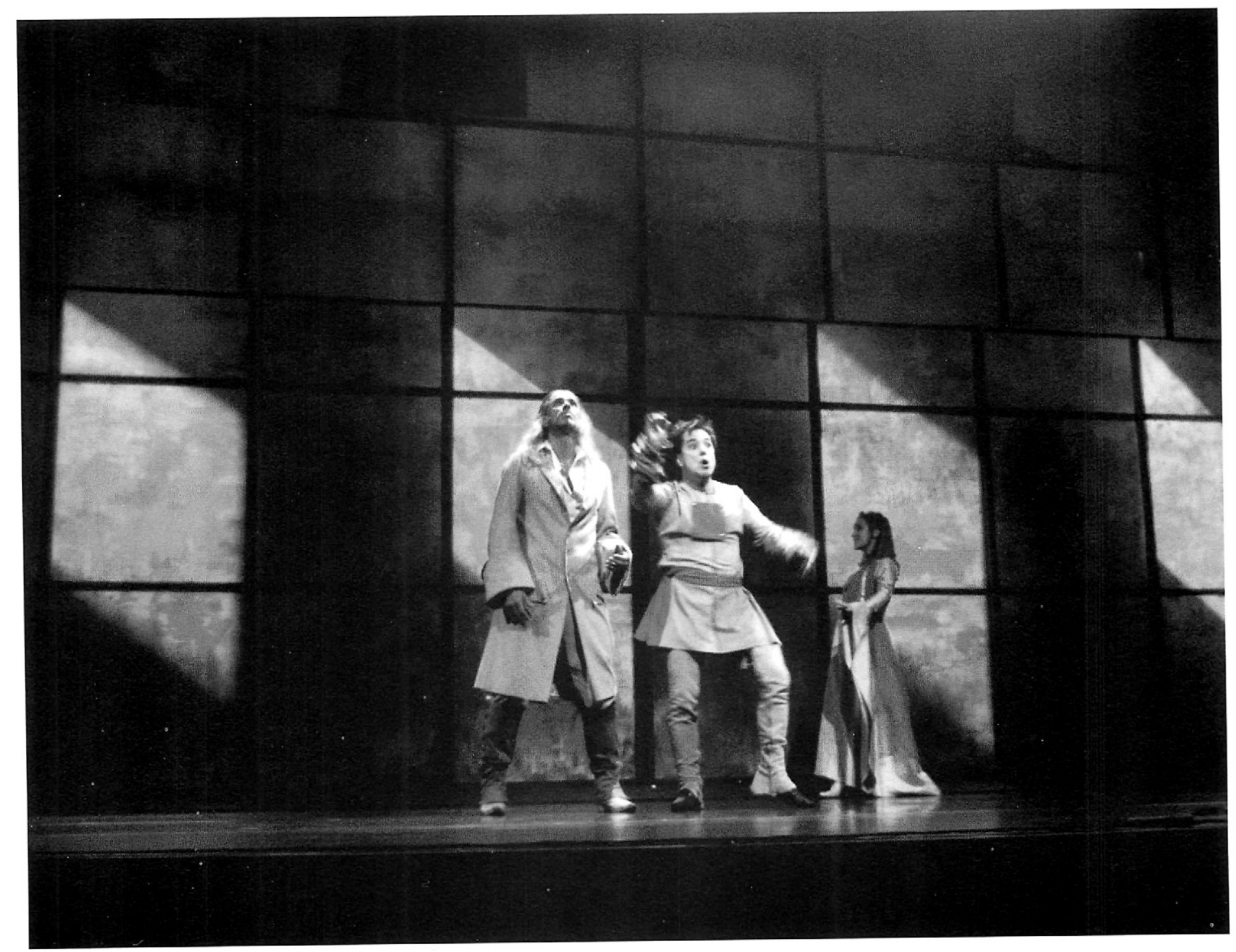




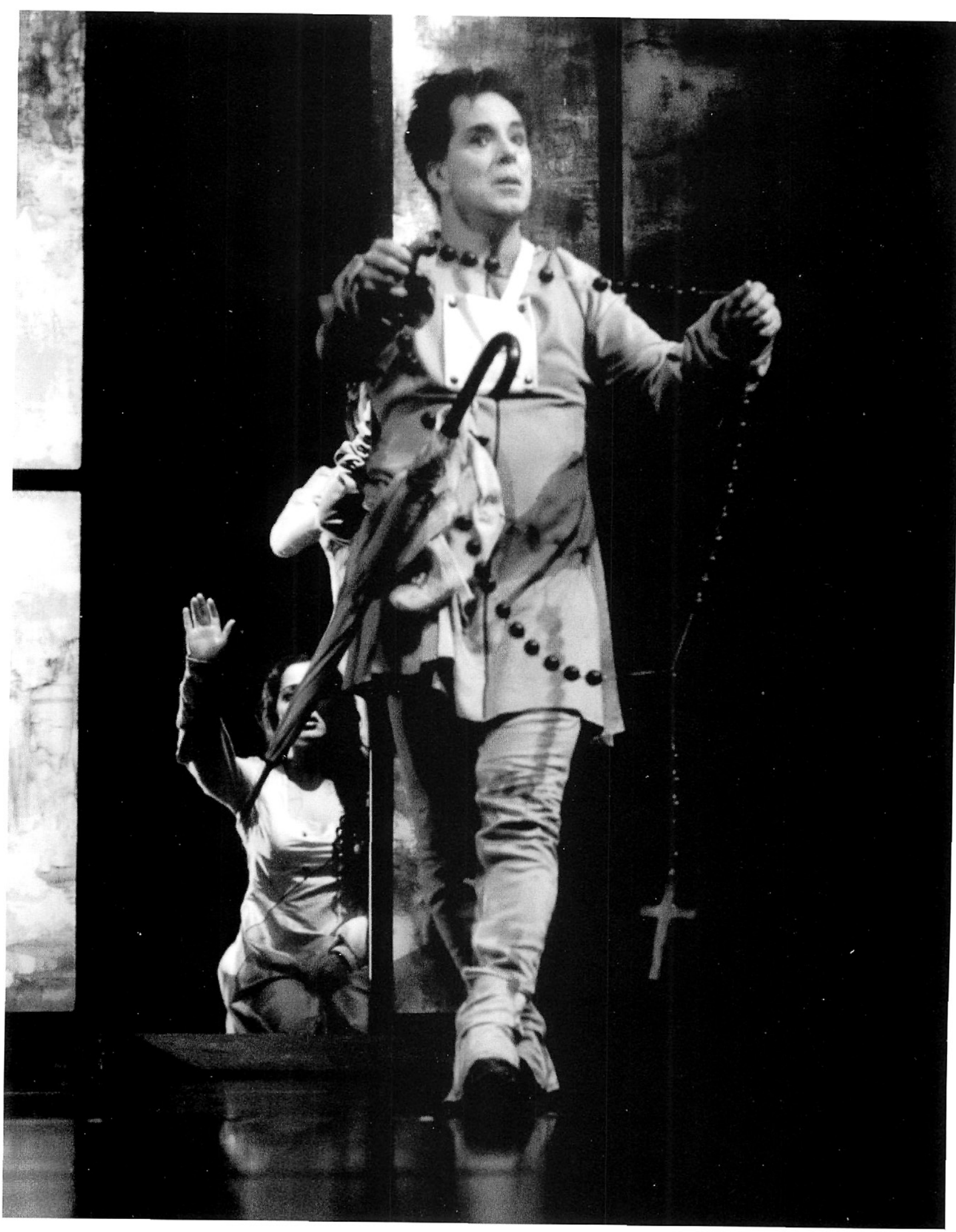

